\title{
A Two-Stage Configuration Method of Integrated Community Energy System Considering Demand Response
}

\author{
Jianfeng Yang ${ }^{1}$, Tianxiang Xie ${ }^{1}$, Chang Zhang ${ }^{1}$, Jie Dong ${ }^{1}$, Jianhao Zhang ${ }^{1}$ and Hequan Zhang ${ }^{1}$ \\ ${ }^{1}$ Economic and Technological Research Institute of State Grid Shaoxing Power Supply Company, Shaoxing, Zhejiang, China
}

\begin{abstract}
The integrated community energy system (ICES) has aroused considerable attention for its low emission and high operating efficiency. The existing configuration methods for ICES with multi-energy sectors ignored the controllable load. In this paper, a two-stage configuration method of ICES is developed to achieve the minimum annual investing and operating cost. At the first stage, the capacities of components in ICES are optimized to minimize the annual investment cost of ICES. At the second stage, the annual operating cost including the electricity and gas purchase costs and the component maintenance cost is minimized to satisfy the energy load. The controllable load under the time-of-use energy price in seasonal typical days is considered in the second stage. Relevant simulations are conducted to validate the effectiveness of the proposed configuration method for ICES. Considering the controllable load, comparative simulations illustrate that the proposed configuration method can significantly reduce the battery investment cost.
\end{abstract}

\section{INTRODUCTION}

The increasing focus on the efficiency of energy sectors and the reduction of greenhouse gas emission in recent years has aroused attention on the integrated energy system (ICES) [1]. ICES, also known as the multi-vector energy system, is comprised of multiple energy sectors, such as electricity, heating, cooling, and gas. ICES aims to achieve the energy cascade utilization and the pollution reduction [2]. Compared with the conventional configuration methods in which each energy sector is planned and operated individually, the ICES can be optimized as a whole system by coordinating all the energy sectors.

Several studies have been carried out to investigate the configuration methods for ICES. The existing configuration methods mainly focus on the energy hub design [3], the ICES configuration with renewable energy uncertainties [4], the energy network configuration [5], and the expansion configuration for ICES [6]. However, the controllable load is not fully considered in these existing researches.

In ICES, one energy supplier purchases the electricity and natural gas from the distributed energy network and sells them to energy consumers. The controllable load of energy consumers, such as HVACs [7], heat pumps [8], and electric refrigerators [9], are sensitive to the energy prices and can adjust their power consumptions for the lower energy cost. Thus, the controllable load should be considered under the timeof-use (TOU) energy prices. However, relevant researches mainly focus on the operation horizon [10], and the controllable load has not been fully investigated in the ICES configuration process.

To address these crucial issues, a two-stage configuration method of integrated community energy system considering demand response is proposed. At the first stage, the annual investment cost is minimized to obtain the capacity of components in the planning horizon. At the second stage, annual operation cost is minimized considering the demand response.

The rest of this paper is organized as follows. The framework of the two-stage planning method for ICES is illustrated in Section 2. Section 3 builds the two-stage planning model for ICES. Section 4 discusses the simulation results, and section 5 gives some remarkable conclusions.

\section{CONFIGURATION METHOD}

The configuration method is to determine the capacity sizes of the ICES components, the modified load profile and the component outputs with the minimum annual cost of investment and operation in the time horizon of $\mathrm{Y}$ year, as shown in Fig. 1.

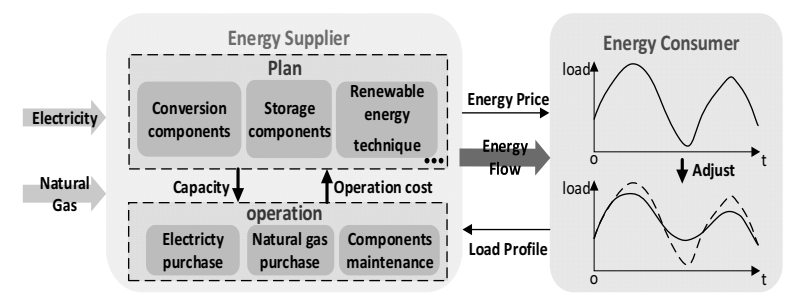

Fig. 1. The framework of the two-stage configuration method.

\footnotetext{
*Corresponding author: yjfwqy@126.com
} 
At the first stage, the capacity of each IECS component is determined with the minimum the annual investment cost based on the given installation limitations. Then the obtained optimal capacities of the IECS components are taken as the constraints at the second stage. The modified load profile and the component outputs are determined with the minimum annual operation cost at the second stage, considering controllable load on the TOU energy price.

\section{CONFIGURATION MODEL}

The annual investment and operation cost is shown in Eq. (1). The annual investment cost $C_{\text {inv }}$ is calculated by Eq. (2). The annual operation cost $C_{\text {opr }}$ is calculated by Eq. (3) considering the natural gas, the electricity purchase cost and the maintenance cost.

$$
\begin{gathered}
C=C_{\text {inv }}+C_{\text {opr }} \\
C_{\text {inv }}=\mathrm{R}\left(\sum_{i \in l} \operatorname{lnv}_{i} C a_{i}\right), \mathrm{R}=\left(\frac{r(1+r)^{y}}{(1+r)^{y}-1}\right) \\
C_{\mathrm{opr}}=\sum_{s=1}^{\mathrm{S}} \mathrm{n}_{s} \sum_{t=1}^{\mathrm{T}}\left(\mathrm{C}_{\text {grid }}(t) P_{\text {grid }}(t)+\right. \\
\left.\mathrm{C}_{\text {gas }} P_{\text {gas }}(t)+\sum_{i \in l} \mathrm{C}_{\mathrm{Mi}} P_{i}(t)\right)
\end{gathered}
$$

where $\operatorname{Inv}_{i}$ is the investment cost of component $i$ per $\mathrm{kW} / \mathrm{kWh} ; C a_{i}$ is the capacity of component $i ; r$ is the discount rate; $y$ is the configuration period; $\mathrm{C}_{\text {gird }}(t)$ and $\mathrm{C}_{\text {gas }}$ are electricity and natural gas price, respectively; $P_{\text {gird }}(t)$ and $P_{\text {gas }}(t)$ are the purchased electricity and gas at time $t$, respectively.

\subsection{Model of ICES}

This paper takes photovoltaic (PV) into account as a clean and low-carbon generation source. The output characteristic of PV is given by Eq. (4).

$$
P_{\mathrm{PV}}(t)=f_{\mathrm{pV}} C_{\mathrm{PV}}\left(\frac{G_{\text {solar }}(t)}{G_{\mathrm{STC}}}\right)
$$

where $P_{\mathrm{PV}}(t)$ is the output power of $\mathrm{PV} ; C_{\mathrm{PV}}$ is the rated capacity of PV; $G_{\text {solar }}(t)$ is the solar radiation.

Working with the waste heat recovery heat boiler (WHRB) makes microturbine (MT) play a significant role in ICES. The output characteristics of MT and WHRB are described by Eq. (5) and Eq. (6), respectively.

$$
\begin{array}{r}
\left\{\begin{array}{c}
P_{\mathrm{MT}}(t)=\eta_{\mathrm{g}} P_{\mathrm{MT}}^{\mathrm{gas}}(t) \\
Q_{\mathrm{MT}}(t)=\eta_{\mathrm{h} 2 \mathrm{p}} P_{\mathrm{MT}}(t)
\end{array}\right. \\
Q_{\mathrm{WHRB}}(t)=\eta_{\mathrm{WHRB}} Q_{\mathrm{MT}}(t)
\end{array}
$$

where $P_{\mathrm{MT}}(t)$ and $Q_{\mathrm{MT}}(t)$ are the electricity and heating outputs of MT at the time $t$, respectively; $P_{\mathrm{MT}}^{\text {gas }}(t)$ the purchased natural gas; $Q_{\mathrm{WHRB}}(t)$ is the heating output of WHRB; $\eta_{\mathrm{g}}$ and $\eta_{\mathrm{WHHB}}$ are the efficiency of MT and WHRB, respectively; and $\eta_{\mathrm{h} 2 \mathrm{p}}$ is the heat to power ratio of MT.

When the MT generates insufficient heat, the deficiency is supplemented by the gas boiler (GB). Characteristics of GB are given by Eq. (7).

$$
P_{\mathrm{GB}}^{\mathrm{h}}(t)=\eta_{\mathrm{GB}} P_{\mathrm{GB}}^{\text {gas }}(t)
$$

where $P_{\mathrm{GB}}^{\text {gas }}(t)$ the natural gas input of gas boiler; $P_{\mathrm{GB}}^{\mathrm{h}}(t)$ the amount of heat output; $\eta_{\mathrm{GB}}$ is the efficiency of GB.

Cooling load in ICES is satisfied by electric refrigerators (ER) as described by Eq. (8).

$$
P_{\mathrm{ER}}^{\mathrm{C}}(t)=\eta_{\mathrm{ER}} P_{\mathrm{ER}}(t)
$$

where $P_{\mathrm{ER}}(t)$ denotes the electricity consumed by ER; $\eta_{\mathrm{ER}}$ is the efficiency of ER; and $P_{\mathrm{ER}}^{\mathrm{C}}(t)$ the cooling energy produced by ER.

The battery (BAT) can act as storage component in ICES. It is assumed that its initial state-of-charge (SOC) is equal to the value of its last charging cycle. The characteristics of BAT are tackled in Eq. (9).

$$
\begin{aligned}
& W_{\mathrm{BAT}}(t)= W_{\mathrm{BAT}}(t-1)\left(1-\rho_{\mathrm{BAT}}\right)+v_{\mathrm{BAT}} P_{\mathrm{BAT}}^{\mathrm{ch}}(t) \eta_{\mathrm{ch}} \\
&-\left(1-\rho_{\mathrm{BAT}}\right) P_{\mathrm{BAT}}^{\text {dis }}(t) \eta_{\mathrm{dis}} \\
& W_{\mathrm{BAT}}(T)= W_{\mathrm{BAT}}(0) \\
& 0 \leq P_{\mathrm{BAT}}^{\mathrm{ch}}(t) \leq \rho_{\mathrm{BAT}} P_{\mathrm{BAT}}^{\mathrm{ch} \cdot \max } \\
& 0 \leq P_{\mathrm{BAT}}^{\mathrm{dis}}(t) \leq\left(1-\rho_{\mathrm{BAT}}\right) P_{\mathrm{ES}}^{\text {dis.max }}
\end{aligned}
$$

where $W_{\mathrm{BATt}}(t)$ is the BAT energy storage; $\sigma_{\mathrm{BAT}}$ is the self-discharge rate of BAT; $P_{\mathrm{BAT}}^{\mathrm{ch}}(t) \mathrm{d} P_{\mathrm{BAT}}^{\text {dis }}(t)$ e charging and discharging power of BAT, respectively; $\eta_{\mathrm{ch}}$ and $\eta_{\text {dis }}$ are the efficiency of charge and discharge, respectively; Binary variable $\rho_{\mathrm{BAT}}$ is introduced to indicate the charging and discharging state; $P_{\mathrm{BAT}}^{\text {ch.max }}$ is the maximum charging.

\subsection{Controllable load}

In the ICES, energy sectors couple with each other through energy conversion components, and the energy supplier provides the TOU energy price to energy consumers. The controllable energy consumers shift their load consumptions from the peak period of energy price to the off-peak period, under the premise of ensuring their comfort levels. The consumers will adjust the controllable load to get their energy cost reduced and this characteristic can help supplier reduce the configuration and operating cost as well. Considering that not all energy load are controllable load, the characteristics of energy consumer is described by Eq. (10) and Eq. (11).

$$
\left\{\begin{array}{c}
\lambda_{\min } L^{\mathrm{e}}(t) \leq P^{\text {out }}(t) \leq \lambda_{\max } L^{\mathrm{e}}(t) \\
\mu_{\min } L^{\mathrm{e}} \leq P^{\text {in }}(t) \leq \mu_{\max } L^{\mathrm{e}}(t) \\
\sum_{t=1}^{T} P^{\text {out }}(t)=\sum_{t=1}^{T} P^{\text {in }}(t)
\end{array}\right.
$$

where $L^{\mathrm{e}}(t)$ is the electrical load; $\lambda_{\min }$ and $\mu_{\min }$ are minimum coefficients of transferring out and transferring in, respectively; $\lambda_{\max }$ and $\mu_{\max }$ are the coefficients of maximum amount transferring out and transferring in, respectively. Eq. (11) indicates that the total energy transferred out is equivalent to the total energy of transferred in. 


\subsection{Constraints}

The energy balance equations for the IECS are given by Eq. (12), Eq. (13), and Eq. (14).

$$
\begin{gathered}
P_{\text {grid }}(t)+P_{\mathrm{PV}}(t)+P_{\mathrm{MT}}(t)+P_{\mathrm{ES}}^{\mathrm{dis}}(t)-P_{\mathrm{ES}}^{\mathrm{ch}}(t) \\
=L^{\mathrm{e}}(t)-\mu P^{\mathrm{out}}(t)+(1-\mu) P^{\text {in }}(t)+P_{\mathrm{ER}}(t) \\
Q_{\mathrm{WHRB}}(t)+Q_{\mathrm{GB}}(t)=L^{\mathrm{h}}(t) \\
P_{\mathrm{ER}}^{\mathrm{c}}(t)=L^{\mathrm{c}}(t)
\end{gathered}
$$

where $L^{\mathrm{h}}(t)$ and $L^{\mathrm{c}}(t)$ are the heating load, cooling load, respectively; binary variable $\mu$ are introduced to indicate the transferring state of controllable load, $\mu=1$ indicates the controllable load is transferring out, otherwise, the controllable load is transferring in. The load profiles during seasonal typical days based on annual load profile are utilized to calculate with less amount.

To solve the problem mentioned above, the mixinteger linear programming method is used in this paper.

\section{CASE STUDY}

The ICES configuration model is realized in the case study under a ten-year configuration horizon. The parameters of each component in the ICES are listed in Table 1.

It is assumed that the electricity price presents the time-of-use character in the configuration period. The natural gas price is $0.3715 \$ / \mathrm{m}^{3}$, which is equal to 0.036 $\$ / \mathrm{kWh}$ (energy content in natural gas is $37.26 \mathrm{MJ} / \mathrm{m}^{3}$ ). Among all the electrical consumers, the controllable consumers account for $30 \%$. The comparison simulations are conducted with and w/o the consideration of controllable electrical consumers.

Table 1. Component parameters.

\begin{tabular}{ccc}
\hline Component & $\begin{array}{c}\text { Minimum Capacity } \\
(\mathrm{kW}, \mathrm{kWh})\end{array}$ & $\begin{array}{c}\text { Maximum Capacity } \\
(\mathrm{kW}, \mathrm{kWh})\end{array}$ \\
\hline MT & 10 & 1000 \\
GB & 50 & 500 \\
EF & 100 & 500 \\
BAT & 0 & 100 \\
WHRB & 0 & 500 \\
PV & 0 & 10 \\
\hline component & Investment cost & Operation cost \\
& $(\$ / \mathrm{kW}, \$ / \mathrm{kWh})$ & $(\$ / \mathrm{kW}, \$ \mathrm{kWh})$ \\
\hline MT & 900 & 0.004 \\
GB & 74 & 0.003 \\
EF & 295 & 0.002 \\
BAT & 209 & 0.0003 \\
WHRB & 74 & 0.002 \\
PV & 3000 & 0 \\
\hline
\end{tabular}

Comparative results about the capacity and cost of ICES are shown in Fig. 2 and Fig. 3, respectively. Compared with the case w/o the consideration of controllable load, the annual investment cost is reduced by $18.20 \%$, and the annual operation cost is $9.47 \times 105$ $\$$ and is reduced by $23.55 \%$. It should be noted that the capacity of battery is significantly decreased from $187.36 \mathrm{kWh}$ to $14.52 \mathrm{kWh}$. The reason is that the controllable electrical consumers provide the alternative energy capacity for the battery to some degree.

In terms of energy management of ICES, the outputs of electrical components in autumn typical day are shown in Fig. 4. It can be observed from Fig. 4 that the controllable load is transferred from peak time to offpeak time, resulting in reduction of electricity purchase cost. MT tends to generate electricity at peak time to reduce electricity cost.

It can be observed from Fig. 4 that the deferrable load is transferred from peak time to off-peak time, resulting in reduction of electricity purchase cost. MT tends to generate electricity at peak time to reduce electricity cost. Fig. 5 and Fig. 6 show the components can satisfy the cooling and heating load in typical days.

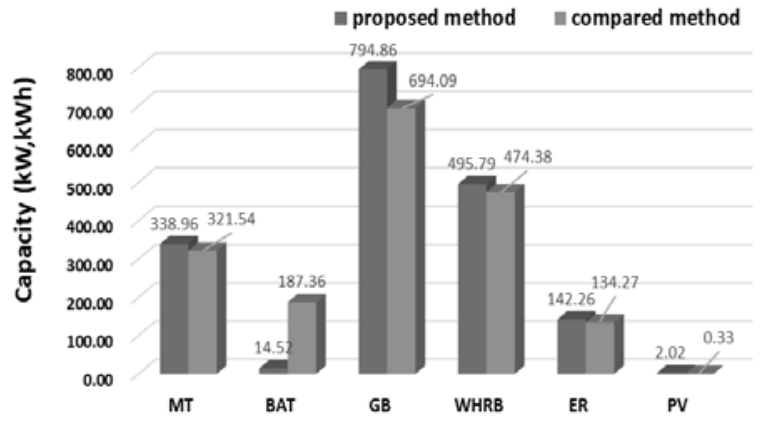

Fig. 2. Comparison of capacity.

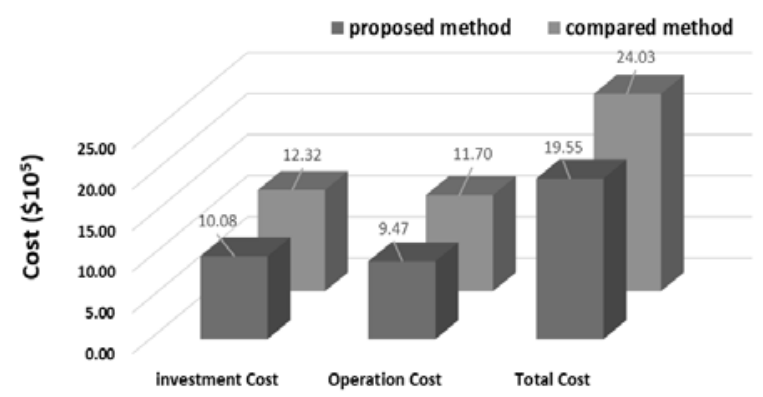

Fig. 3. Comparison of cost.

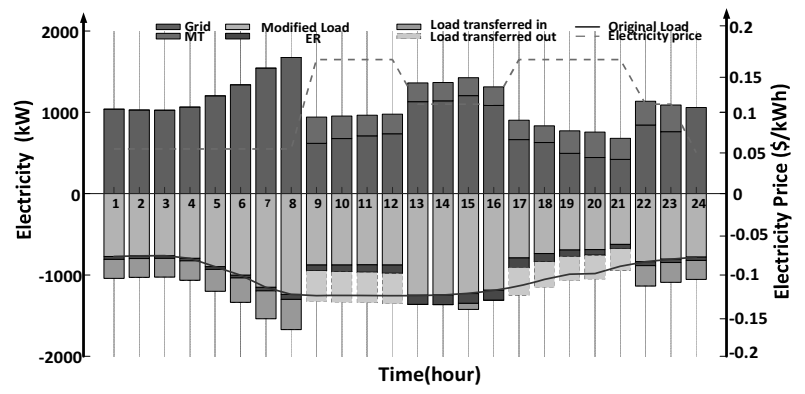

Fig. 4. Autumn electricity energy management.

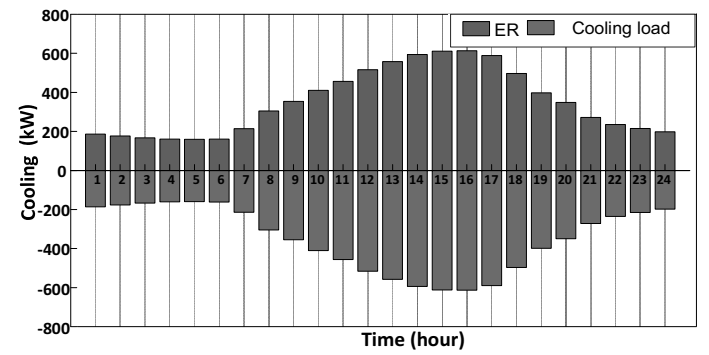

Fig. 5. Summer cooling energy management. 


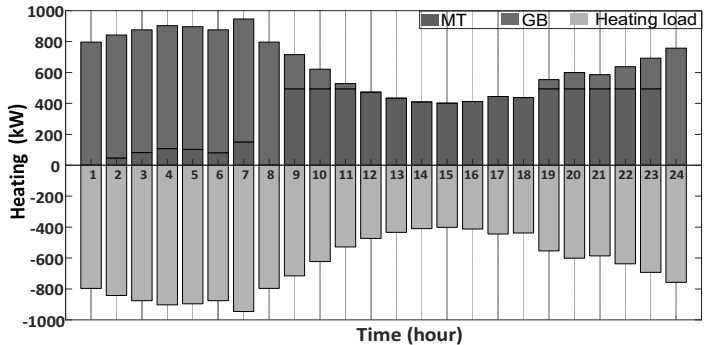

Fig. 6. Winter heating energy management.

\section{CONCLUSION}

This paper proposed a two-stage configuration method to solve the ICES configuration problem considering the controllable load. Results show that the proposed method can reduce the annual investment cost and annual operation cost remarkably, especially the investment cost of battery.

\section{References}

1. Shao C, Ding Y, Wang J, Song Y. Modeling and integration of flexible demand in heat and electricity integrated energy system. IEEE Transactions on Sustainable Energy. 2018;9:361-70.

2. Geidl M. Integrated Modeling and Optimization of Multi-Carrier Energy Systems: ETH; 2007.

3. Wang Y, Zhang N, Zhuo Z, Kang C, Kirschen D. Mixed-integer linear programming-based optimal configuration planning for energy hub: Starting from scratch. Applied energy. 2018;210:1141-50.

4. Wang Y, Zhang N, Zhuo Z, Kang C, Kirschen D. Mixed-integer linear programming-based optimal configuration planning for energy hub: Starting from scratch. Applied energy. 2018;210:1141-50.

5. Wang Y, Zhang N, Zhuo Z, Kang C, Kirschen D. Mixed-integer linear programming-based optimal configuration planning for energy hub: Starting from scratch. Applied energy. 2018;210:1141-50.

6. Qiu J, Dong ZY, Zhao JH, Meng K, Zheng Y, Hill DJ. Low carbon oriented expansion planning of integrated gas and power systems. IEEE Transactions on Power Systems. 2015;30:1035-46.

7. Kusiak A, Li M, Tang F. Modeling and optimization of HVAC energy consumption. Applied Energy. 2010;87:3092-102.

8. Franco A, Fantozzi F. Experimental analysis of a self consumption strategy for residential building: The integration of PV system and geothermal heat pump. Renewable Energy. 2016;86:1075-85.

9. Franco A, Fantozzi F. Experimental analysis of a self consumption strategy for residential building: The integration of PV system and geothermal heat pump. Renewable Energy. 2016;86:1075-85.

10. Wang D, Zhou Y, Jia H, Wang C, Lu N, Sui P-C, et al. An energy-constrained state priority list model using controllable electrolyzers as a load management mechanism. Applied Energy. 2016;167:201-10. 